\section{Elevated post-prandial free fatty acids are associated with cardiac sympathetic overactivity in Type II diabetic patients}

\begin{abstract}
To the Editor: Post-prandial hyperglycaemia and possibly other metabolic abnormalities have been suggested to be potential risk factors for sudden death since they prolong the QTc interval in diabetic patients [1]. It has also been shown that increased plasma free fatty acid (FFA) concentrations stimulate the cardiac autonomic nervous system in healthy subjects [2]. Due to the strong metabolic link between FFA and triglyceride concentrations, one can not exclude that increased post-prandial plasma FFA concentrations might have a role on modulating cardiac autonomic nervous system in Type II (non-insulin-dependent) diabetic patients. So far, no study has evaluated the possible relationship between changes in post-prandial plasma FFA concentrations and the cardiac autonomic nervous system in Type II diabetic patients. For these reasons 25 Type II diabetic patients (mean age: $66.3 \pm 4.8$ years; $\mathrm{HbA}_{1 \mathrm{c}}=7.3 \pm 0.3 \%$ ) treated with glibenclamide ( $5 \mathrm{mg}$ twice daily) were submitted during $24 \mathrm{~h}$ to power spectral analysis of heart rate variability (HRV) for assessing cardiac autonomic nervous system activity at fasting, before and after lunch. HRV was calculated by autoregressive model as previously reported [3]. In such a model low frequency/high frequency (LF/HF ratio) is considered a good index of sympathetic/parasympathetic balance at cardiac levels [2]. Respiratory frequency was also calculated [2]. A standardized fat lunch made up by $\pm 760 \mathrm{kcal}$ divided in proteins (12.3\% of energy), fat (59.2\% of energy) and carbohydrate $(28.5 \%$ of energy) was made to investigate the post-prandial metabolic changes. In particular, the following fats were in the diet: $50 \mathrm{~g}$ of fat, $29.4 \mathrm{~g}$ of saturated fat and $50 \mathrm{~g}$ of cholesterol. The meals were prepared in one batch and consumed under the supervision of a nurse. The degree of oxidative stress in serum was measured as the reaction of malondialdehyde with thiobarbituric acid reactive substance [3]. Furthermore, the plasma total antioxidant capacity was estimated by the 2,2-azinobis-3-ethylbenzothiazoline-6-sulfonic acid radical cation decol-orization assay, with use of the Trolox (St. Louis, Mich., USA) standard [4]. Plasma glucose (glucose autoanalyzer, Beckman Coulter, Fullerton, Calif., USA) tryglicerides [Peri-
\end{abstract}

Published online: 16 November 2002

(C) Springer-Verlag 2002 decrome, Roche, Basel, Switzerland $(\mathrm{CH})]$ and FFA (routine microfluormetric method) concentrations [5] were also assessed. Furthermore, all patients had all metabolites assessed at fasting as well as at pre-prandial and 2-h post-prandial conditions. All subjects gave their informed consent before participating in the study which was approved by the ethics committee of our institutions.

All data are shown as means $\pm \mathrm{SD}$. Changes in plasma metabolites and LF/HF ratio were calculated as the difference between post-prandial and pre-prandial values. Analysis of variance (ANOVA) was used to calculate differences among the different experimental conditions. A $p$ value of less than 0.05 was shown, Scheffe's test was also applied to analyse which intervention most influenced the overall difference between groups. Partial correlations were used to examine the association between the two variables independently of covariates. A $p$ value of 0.05 was chosen as the level of significance.

Despite oral hypoglycaemic agents, post-prandial glucose and FFA concentrations were more augmented than in preprandial and fasting conditions (Table 1). Similarly, analysis of HRV parameters provided evidence that LF to HF ratio was more elevated in post-prandial than in pre-prandial and fasting conditions (Table 1). Independent of changes in plasma glucose concentrations, changes in post-prandial FFA correlated with changes in the post-prandial $\mathrm{LF} / \mathrm{HF}$ ratio ( $r=0.57$; $p<0.001)$ and with changes in plasma TBARS $(r=0.49$; $p<0.01)$ and TEAC $(r=0.46 ; p<0.02)$ concentrations. After adjustment for changes in plasma glucose concentrations, changes in the LF/HF ratio were also correlated with the changes in plasma TBARS $(r=0.52 ; p<0.003)$ and TEAC $(r=0.50$; $p<0.002)$.

Our study shows that increased post-prandial plasma FFA concentrations are associated with an enhanced degree of oxidative stress and of LF/HF ratio, an index of cardiac sympathovagal balance. Such data seems particularly relevant for explaining the relationship among plasma FFA, oxidative stress and sudden death in Type II diabetic patients. In fact, it has been shown that increased plasma FFA concentrations are a pro-oxidant factor [5] and that unbalanced sympathetic or parasympathetic tone seems linked to a degree of oxidative stress [6]. Furthermore, plasma concentrations are responsible for many cases of sudden death [7] despite the absence of documented pre-existing heart disease [7]. Chronic administration of an antioxidant agent such vitamin $\mathrm{E}$ has been shown to lower oxidative stress in Type II diabetic patients and to re-balance cardiac sympathetic activity [6], the latter phenomenon being associated with a decline in plasma catecholamine concentrations [6]. Notwithstanding, the concept that antioxidants are 
Table 1. Effects of fat-meal on metabolic parameters and cardiac sympatho-vagal balance (LF/HF ratio)

\begin{tabular}{|c|c|c|c|c|c|}
\hline & Fasting & $p$ & Pre-prandial & $p$ & 2-h post-prandial \\
\hline Plasma glucose (mmol/l) & $8.1 \pm 0.6^{b}$ & NS & $8.5 \pm 0.4$ & 0.005 & $9.8 \pm 0.1$ \\
\hline Plasma triglyceride $(\mathrm{mmol} / \mathrm{l})$ & $1.4 \pm 0.3^{\mathrm{a}}$ & NS & $1.6 \pm 0.1$ & 0.01 & $2.8 \pm 0.2$ \\
\hline Plasma TEAC $(\mathrm{mmol} / \mathrm{l})$ & $1.5 \pm 0.4^{b}$ & NS & $1.3 \pm 0.6$ & 0.005 & $0.40 \pm 0.04$ \\
\hline Plasma TBARS (nmol MDA/L plasma) & $0.69 \pm 0.01^{\mathrm{b}}$ & NS & $0.67 \pm 0.03$ & 0.005 & $2.7 \pm 0.2$ \\
\hline $\mathrm{LF} / \mathrm{HF}$ ratio & $3.2 \pm 0.3^{c}$ & NS & $3.5 \pm 0.2$ & 0.001 & $5.3 \pm 0.2$ \\
\hline
\end{tabular}

Fasting vs 2-h post-prandial:

${ }^{\mathrm{a}} p<0.01 ; \quad{ }^{\mathrm{b}} p<0.005 ; \quad{ }^{\mathrm{c}} p<0.001$

particularly helpful to lower cardiovascular diseases has been seriously questioned by the Heart Protection Study [8] that did not support such a hypothesis. We should also emphasize the fact that our study had three limitations: (i) there was no carbohydrate enriched meal to check for a rise in FFA; (ii) no insulin concentrations were measured, which are an important modifier of the sympathetic tone; (iii) study design and statistical analysis did not allow us to establish a cause and effect conclusion. Furthermore, it should be also pointed out that increased plasma FFA concentrations might exert their negative impact on the cardiac autonomic nervous system independently of the degree of oxidative stress. In fact, it has been shown that increased plasma FFA concentrations have an indirect pressor effect on vascular bed, inhibit $\mathrm{Na} / \mathrm{K}$ ATPase pump and exert a toxic activity mainly due to their detergent effect at plasma membrane levels. Among such hypotheses, a neural pressor response to increased FFA concentrations is the most suitable hypothesis, since a previous investigation showed a rise in plasma catecholamine concentrations during the infusion of Lipid emulsion and heparin in healthy subjects [2] as well as a partial protection from such phenomenon after propranolol administration [2].

In conclusion, our data allow us to conclude that increased post-prandial FFA concentrations are associated with a rise in degree of oxidative stress and a neural pressor response which have a negative impact on cardiac sympathetic and parasympathetic balance. Such a latter event might provide further pathophysiological insight for explaining the relationship between increased plasma concentrations FFA and sudden death in Type II diabetic patients.

D. Manzella, R. Grella, R. Marfella, D. Giugliano, G. Paolisso
All results are means $\pm \mathrm{SD}$; all parameters are considered at fasting condition; TEAC, Rolox-equivalent antioxidant capacity; TBARS, thiobarbituric acid-reactive substances.

\section{References}

1. Heine RJ, Dekker JM (2002) Beyond postprandial hyperglycaemia: metabolic factors associated with cardiovascular disease. Diabetologia 45: 461-475

2. Paolisso G, Manzella D, Rizzo MR et al. (2000) Elevated plasma fatty acid concentrations stimulate the cardiac autonomic nervous system in healthy subjects. Am J Clin Nutr 72: 723-730

3. Griesmaker A, Kondhauser M, Andert SE et al. (1995). Enhanced serum levels of thiobarbituric-acid reactive substance in diabetes mellitus. Am J Med 98:469-475

4. Pellegrini N, Re R, Yang M, Rice-Evans C (1998) Screening of dietary carotenoids and carotenoids-rich fruit extracts for antioxidant activities applying 2,2'-azinobis(3-ethylenebenzothiazoline-6-sulfonic acid radical cation decolorization assay. Methods Enzymol 299: 379-389

5. Paolisso G, Gambardella A, Tagliamone MR et al. (1996) Does free fatty acid infusion impair insulin action also trough an increase in oxidative stress? J Clin Endocrinol Metab 81: 4244-4248

6. Manzella D, Barbieri M, Ragno E, Paolisso G (2001) Chronic administration of pharmacologic doses of vitamin $\mathrm{E}$ improves the cardiac autonomic nervous system in patients with type 2 diabetes. Am J Clin Nutr 73: 1052-1057

7. Jouven X, Charles MA, Desnos M, Ducimetiere P (2001) Circulating nonesterified fatty acid as a predictive risk factor for sudden death in the population. Circulation 104: 756-761

8. Heart Protection Study Group Collaborative Group (2002) Heart Protection Study of antioxidant vitamin supplementation in 20536 high-risk individuals: a randomised placebocontrolled trial. Lancet 360: 23-33

Corresponding author: G. Paolisso, Department of Geriatric Medicine and Metabolic Diseases, Internal Medicine IV, Piazza Miraglia 2, 80138 Napoli, Italy. E-mail: giuseppe.paolisso@unina2.it 